\title{
AlGaN/GaN Heterojunction Bipolar Transistor
}

\author{
L. S. McCarthy, P. Kozodoy, M. J. W. Rodwell, Senior Member, IEEE, \\ S. P. DenBaars, Member, IEEE, and U. K. Mishra, Fellow, IEEE
}

\begin{abstract}
We demonstrate the first GaN bipolar transistor. An AlGaN/GaN HBT structure was grown by MOCVD on $c$-plane sapphire substrate. The emitter was grown with an $\mathrm{Al}_{0.1} \mathbf{G a}_{0.9} \mathbf{N}$ barrier to increase the emitter injection efficiency. $\mathrm{Cl}_{2} \mathrm{RIE}$ was used to pattern the emitter mesa, and selectively regrown base contact pads were implemented to reduce a contact barrier associated with RIE etch damage to the base surface. The current gain of the devices was measured to be as high as three with a base width of $200 \mathrm{~nm}$. DC transistor characteristics were measured to $30 \mathrm{~V} V_{\mathrm{CE}}$ in the common emitter configuration, with an offset voltage of $5 \mathrm{~V}$. A gummel plot and base contact characteristics are also presented.
\end{abstract}

\section{INTRODUCTION}

$\mathbf{F}$ OR several years, III-Nitride technology has been advancing rapidly. Interest in this material system has been driven by advances in optical devices such as blue lasers and LED's [1], and high-power, high-frequency transistors. The development of the AlGaN/GaN HBT is motivated both by the success of AlGaN/GaN HEMT's which have demonstrated record figures for high power microwave performance [2], [3], and the inherent advantages of HBT's. Bipolar transistors potentially have more uniform threshold voltages, higher linearity and higher current densities than FET's. Transistors with $\mathrm{GaN}$ emitters grown on $\mathrm{SiC}$ base/collector structures have demonstrated high differential gain at high temperatures [4] and GaN/AlGaN phototransistors without a base contact have also shown high gain [5]. Difficulties with p-type GaN however, have hampered research of $\mathrm{AlGaN} / \mathrm{GaN}$ bipolar transistors. High base dopant concentration is required to achieve low output conductance, but may increase recombination rates and reduce electron velocity. Additionally, high frequency devices will require self-aligned emitter-base lithography and thick contact regions to reduce base access resistance. With its wide bandgap, $3.4 \mathrm{eV}, \mathrm{GaN}$ offers a high breakdown base-collector junction with a shorter collector length than in conventional material systems. Predicted maximum electron velocities of $2 \times 10^{7} \mathrm{~cm} / \mathrm{s}$ [6] in n-type $\mathrm{GaN}$ with breakdown voltages as high as $2 \times 10^{6} \mathrm{~V} / \mathrm{cm}$ lead to a Johnson's figure of merit, $\left(E_{\mathrm{bk}} \cdot v_{\mathrm{sat}} / \pi\right)^{2}$ of $1.6 \times 10^{26}(\mathrm{~V} / \mathrm{sec})^{2}, 282$ times that of silicon. Furthermore linear extrapolation from the AIN bandgap suggests that a $10 \%$ grade in aluminum composition over $2000 \AA$ leads to quasifields in the base of $1.3 \times 10^{4} \mathrm{~V} / \mathrm{cm}$, substantially reducing base transit time.

Manuscript received October 26, 1998; revised February 4, 1999. This work was funded by a grant from the U.S. Office of Naval Research (ONR).

The authors are with the Department of Electrical and Computer Engineering, the University of California, Santa Barbara, CA 93106 USA.

Publisher Item Identifier S 0741-3106(99)05051-X.

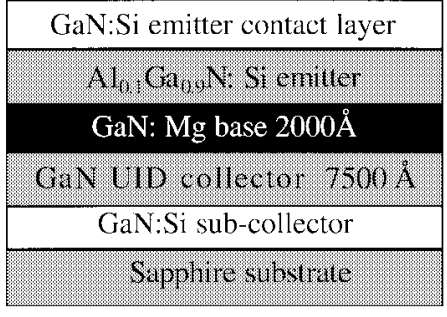

Fig. 1. Layer structure for AlGaN/GaN HBT grown by MOCVD on sapphire.

In this letter we report the first $\mathrm{AlGaN} / \mathrm{GaN}$ HBT. The emitter contact was used as a mask for the emitter mesa etch, and additional base material was re-grown to bury the damaged base surface and reduce extrinsic base resistance.

\section{Device Structure}

The layer structure for these devices was grown by MOCVD on $C$-plane sapphire. An n-type ( $\mathrm{Si}$ doped) subcollector is followed by an unintentionally doped (UID) n-type GaN collector, a p-type (Mg doped) $\mathrm{GaN}$ base, and an n-type ( $\mathrm{Si}$ doped) $\mathrm{Al}_{0.1} \mathrm{Ga}_{0.9} \mathrm{~N}$ emitter (see Fig. 1). The $5000 \AA$ emitter was doped $5 \times 10^{18} \mathrm{~cm}^{-3}$ while the base layer was $2000 \AA$ of $\mathrm{Mg}$ doped $\mathrm{GaN}$ with an acceptor concentration of $1 \times 10^{19}$ $\mathrm{cm}^{-3}$. Magnesium is a deep acceptor in $\mathrm{GaN}, 160 \mathrm{meV}$ from the valence band edge, leading to incomplete ionization and an expected carrier density of $5 \times 10^{17} \mathrm{~cm}^{-3}$. The resistivity of p-type material grown under similar conditions is $1.5 \Omega \cdot \mathrm{cm}$ resulting in sheet resistivities of $75 \mathrm{k} \Omega$ /square for a $2000 \AA$ base. The background donor concentration in the collector is on the order of $5 \times 10^{16} \mathrm{~cm}^{-3}$.

\section{DEVICE FABRICATION}

The transistors had emitter lengths of 20, 50, and $100 \mu \mathrm{m}$ and widths varying from 1 to $3 \mu \mathrm{m}$. Some larger devices $(20 \mu \mathrm{m} \times 20 \mu \mathrm{m})$ were also patterned. After growth, a tungsten emitter contact was deposited and subsequently used as a mask for the $\mathrm{Cl}_{2}$ RIE emitter mesa etch (see Fig. 2). Electrical measurements were used to determine when the base layer had been accessed. SiN was then blanket deposited by PECVD and patterned with an $\mathrm{SF}_{6}$ RIE to form an emitter capping layer. Because the contacts to p-type $\mathrm{GaN}$ are sensitive to underlying damage, base regrowth was used to bury surface damage caused by the emitter etch (see Fig. 3). An anneal at $950{ }^{\circ} \mathrm{C}$ in an $\mathrm{N}_{2}$ ambient was used to re-activate $\mathrm{Mg}$ dopants passivated by hydrogen in the MOCVD reactor [7]. Once activated, base contacts were deposited on the regrown 

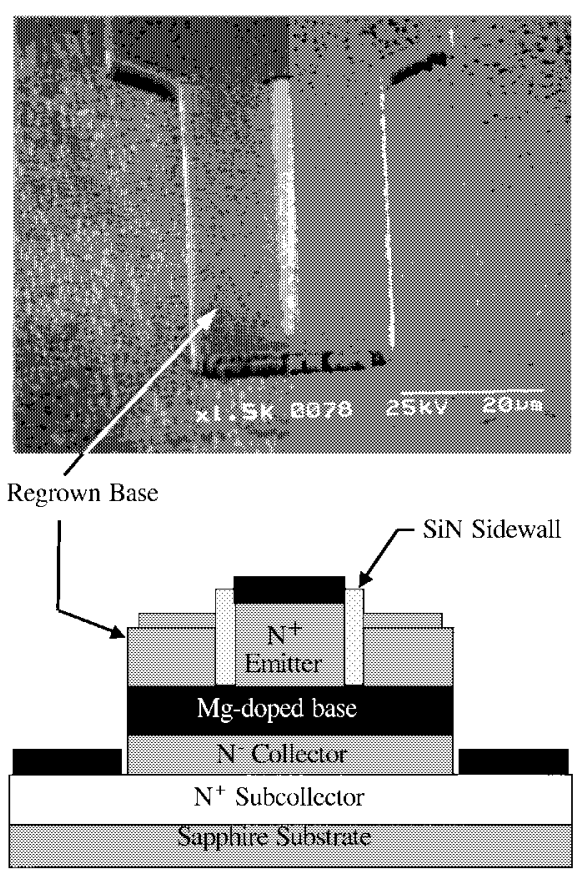

Fig. 3. Cross section and SEM image of completed device.

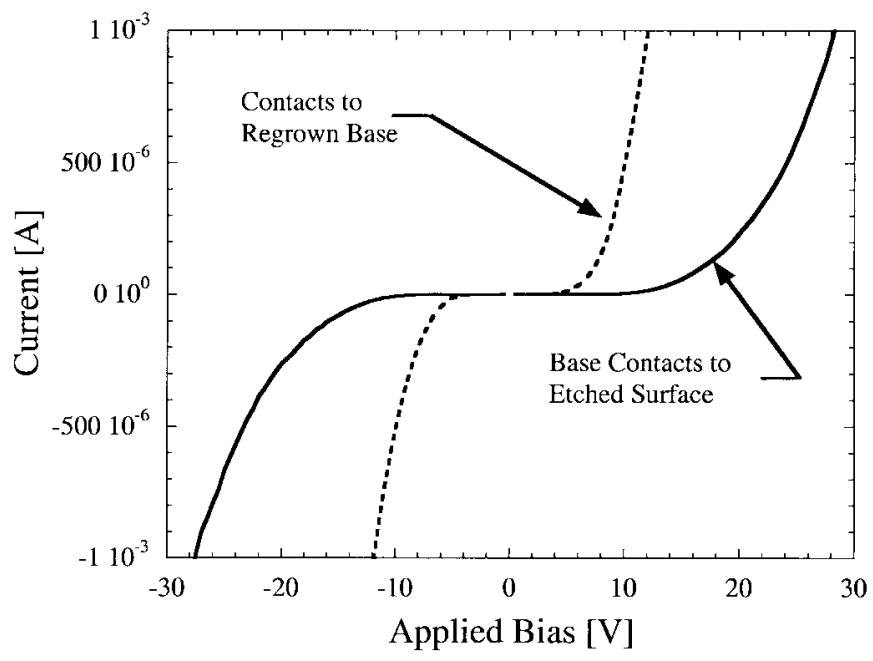

Fig. 2. Current-voltage $(I-V)$ characteristics for p-base contacts. The dashed line was measured on base layer TLM patterns near the transistors presented, while the solid line was measured on a separate sample where contacts were applied to the base layer as etched-without regrowth.

material and the top of the SiN cap was removed to expose the emitter contact. The separation from the edge of the emitter mesa to the base contacts was measured to be $1 \mu \mathrm{m}$. To contact the $\mathrm{Si}$ doped subcollector, a base mesa was defined by $\mathrm{Cl}_{2} \mathrm{RIE}$ and $\mathrm{Al} / \mathrm{Au}$ ohmic contacts were deposited. The contacts were then annealed and device measurements were made.

\section{Electrical Characterization}

An HP 4145 semiconductor analyzer was used to perform the electrical characterization of these devices which were measured in the common emitter configuration. Gummel plots were also taken and are shown with $V_{\mathrm{CB}}=0$. Common emitter characteristics of a $1 \mu \mathrm{m} \times 20 \mu \mathrm{m}$ device (see Fig. 4) as well as a Gummel plot on a $20 \mu \mathrm{m} \times 20 \mu \mathrm{m}$ device (see

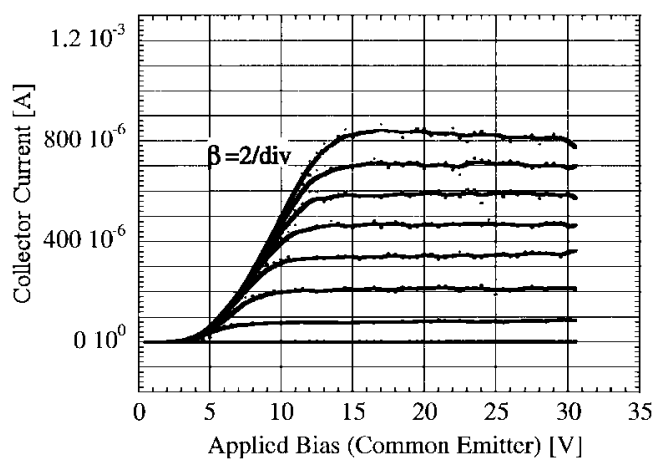

Fig. 4. Common emitter characteristics of $1 \mu \mathrm{m} \times 20 \mu \mathrm{m}$ HBT. Base current was stepped from 0 to $350 \mu \mathrm{A}$ in $50 \mu \mathrm{A}$ steps. The source of the apparent noise in these curves is unknown.

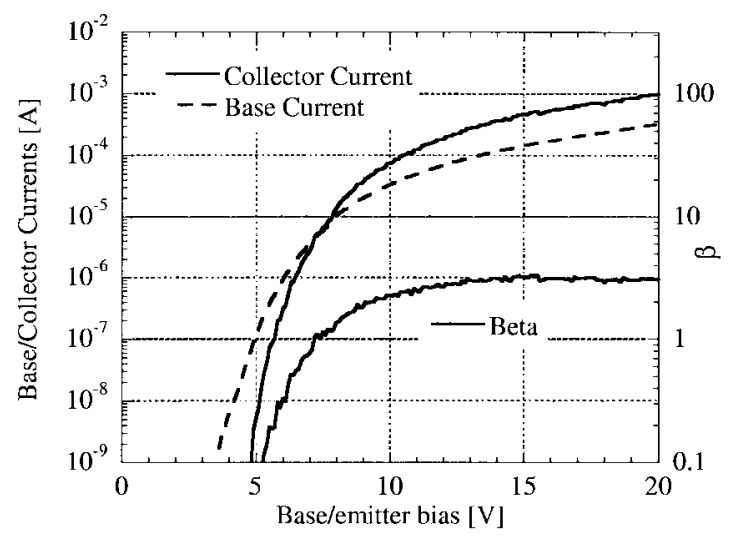

Fig. 5. Gummel plot of $20 \mu \mathrm{m} \times 20 \mu \mathrm{m}$ HBT with $V_{\mathrm{CB}}=0$.

Fig. 5) indicate a current gain $\left(I_{C} / I_{B}\right)$ of 3 . The Early voltage indicated by the common emitter characteristics was too high to measure accurately. An unexpectedly high $(5 \mathrm{~V}) V_{\mathrm{CE}}$ offset was observed in the collector current. Preliminary investigation suggests that the high offset voltage may be linked to the poor quality of the base contacts. Collector currents were on the order of $1 \mathrm{~mA}$ (see Fig. 5), however current density calculations are unreliable because of current crowding effects at the emitter/base junction.

Although not measured for these devices, the expected base sheet resistance inferred from measurements taken on similar material is $75 \mathrm{k} \Omega /$ square and the base resistance due the bulk p-GaN $3.75 \mathrm{k} \Omega$ for the $20 \mu \mathrm{m}$ devices and $750 \Omega$ for the $100 \mu \mathrm{m}$ devices. Due to the poor base contact quality (see Fig. 3) the voltage drop across the contact is thought to dominate the base resistance.

\section{CONCLUSION}

The AlGaN/GaN HBT has demonstrated a current gain of three and common emitter mode operation to $30 \mathrm{~V}$. The low current gains observed may be the result of a combination of a thick base layer, $2000 \AA$, surface recombination, and a high recombination rate in the base/emitter junction. Although the base resistance of these layers was not measured, bulk resistivities measured on material grown under similar conditions suggest a sheet resistance of $75 \mathrm{k} \Omega /$ square. Although poor contacts are currently thought to dominate the base resistance, 
improved surface preparation and material quality are expected to reduce both contact and bulk resistivities. The use of base regrowth reduced the voltage barrier associated with the base contacts from $10 \mathrm{~V}$ to $5 \mathrm{~V}$.

The high-power, high-frequency operation of $\mathrm{AlGaN} / \mathrm{GaN}$ HEMT's [2], [3] has demonstrated the advantages of GaN based material systems for high performance microwave applications. Although significant technological barriers exist to the progress of the AlGaN/GaN HBT, the development of this technology is warranted as it combines the advantages of the wide bandgap and high electron velocity inherent in $\mathrm{GaN}$ and the unique physical attributes of the HBT.

\section{REFERENCES}

[1] S. Nakamura and G. Fasol, The Blue Laser Diode: GaN Based Light Emitters and Lasers. New York: Springer-Verlag, 1997.
[2] S. T. Sheppard, K. Doverspike, W. L. Pribble, S. T. Allen, J. W. Palmour, L. T. Kehias, and T. J. Jenkins, "High power microwave GaN/AlGaN HEMT's on silicon carbide," in 56th Annu. Device Res. Conf. Dig. (Late News), 1998.

[3] Y. F. Wu, B. P. Keller, P. Fini, S. Keller, T. J. Jenkins, L. T. Kehias, S. P. Denbaars, and U. K. Mishra, "High Al-content AlGaN/GaN MODFET's for ultrahigh performance," IEEE Electron Device Lett. vol. 19, pp. 50-53, Feb. 1998.

[4] J. Pankove, S. S. Chang, H. C. Lee, R. J. Molnar, T. D. Moustakas, and B. Van Zeghbroeck, "High-temperature $\mathrm{GaN} / \mathrm{SiC}$ heterojunction bipolar transistor with high gain," in IEDM Tech. Dig., 1994, pp. 389-392.

[5] Y. Wei, T. Nohava, S. Krishnankutty, R. Torreano, S. McPherson, and H. Marsh, "High gain GaN/AlGaN heterojunction phototransistor," Appl. Phys. Lett., vol. 73, no. 7, pp. 978-980, 1998.

[6] U. V. Bhapkar and M. S. Shur, "Monte Carlo calculation of velocityfield characteristics of wurtzite GaN," J. Appl. Phys., vol. 82, no. 4, pp. 1649-1655, 1997.

[7] S. Nakamura, N. Iwasa, M. Senoh, and T. Mukai, "Hole compensation mechanism of p-type GaN films," Jpn. J. Appl. Phys., vol. 31, no. 5A, pp. 1258-1266, 1992. 